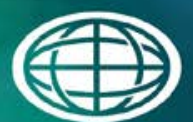

Savannah River

National Laboratory ${ }^{m}$

OPERATED BY SAVANNAH RIVER NUCLEAR SOLUTIONS

\title{
Compositions of Simulated LAW Glasses Saturated with Sulfur at Various \\ Temperatures
}

K. M. Fox

W. T. Riley

April 2018

SRNL-STI-2018-00071, Revision 0 
SRNL-STI-2018-00071

Revision 0

\section{DISCLAIMER}

This work was prepared under an agreement with and funded by the U.S. Government. Neither the U.S. Government or its employees, nor any of its contractors, subcontractors or their employees, makes any express or implied:

1. warranty or assumes any legal liability for the accuracy, completeness, or for the use or results of such use of any information, product, or process disclosed; or

2. representation that such use or results of such use would not infringe privately owned rights; or

3. endorsement or recommendation of any specifically identified commercial product, process, or service.

Any views and opinions of authors expressed in this work do not necessarily state or reflect those of the United States Government, or its contractors, or subcontractors.

\section{Printed in the United States of America \\ Prepared for U.S. Department of Energy}


Keywords: Low activity waste, glass, sulfur, Hanford, WTP

Retention: Permanent

\section{Compositions of Simulated LAW Glasses Saturated with Sulfur at Various Temperatures}

K. M. Fox

W. T. Riley

April 2018

Prepared for the U.S. Department of Energy under 


\section{REVIEWS AND APPROVALS}

\section{AUTHORS:}

K. M. Fox, Waste Form Processing Technologies

W. T. Riley, Environmental Stewardship Directorate Operations

TECHNICAL REVIEW:

D. L. McClane, Immobilization Technology, Reviewed per E7 2.60

Date
Date

Date

APPROVAL:

C. C. Herman, Director, Waste Form Processing Technologies

Date 


\section{ACKNOWLEDGEMENTS}

The authors thank Courtney Burckhalter, Holly Hall, Kandice Miles, and Kim Wyszynski at Savannah River National Laboratory for their skilled assistance with the laboratory analyses described in this report. SRNL Analytical Development staff performed the cesium concentration measurements. The authors thank Tongan Jin at the Pacific Northwest National Laboratory for helpful discussions and review of these data and report. Funding from the U.S. Department of Energy Office of River Protection Waste Treatment and Immobilization Plant Project through Inter-Entity Work Order M0SRV00101 as managed by Albert A. Kruger is gratefully acknowledged. 


\section{EXECUTIVE SUMMARY}

In this report, the Savannah River National Laboratory (SRNL) provides chemical analysis for several simulated low-activity waste (LAW) glass compositions, as well as chemical analysis of the wash solutions resulting from the preparation of these glasses. The Pacific Northwest National Laboratory (PNNL) selected and fabricated these glasses as part of a study on sulfur retention in glasses as a function of melting temperature.

Chemical analyses were performed on a representative sample of each of the study glasses to allow for comparisons with the targeted compositions. Three dissolution techniques, sodium peroxide fusion, lithium metaborate fusion, and potassium hydroxide fusion, were used for preparing each of the glass samples for analysis. Each of the samples was analyzed twice for each element of interest by Inductively Coupled Plasma - Atomic Emission Spectroscopy (ICP-AES) or Ion Chromatography (IC). Cesium concentrations were measured a single time by Inductively Coupled Plasma - Mass Spectroscopy (ICP-MS). A glass standard was also measured as a check of the performance of the analytical instruments. Average concentrations of each of the glass components were determined and reported.

Chemical analyses were also performed on a representative sample of each of the wash solutions resulting from the preparation of the sulfur saturated melt (SSM) versions of the study glasses. The samples were analyzed in duplicate for each element of interest by ICP-AES or IC. Cesium concentrations were measured once by ICP-MS. Average concentrations of each analyte were determined and reported.

These results will be used by PNNL in the development of improved property/composition models for LAW glass production at Hanford. 


\section{TABLE OF CONTENTS}

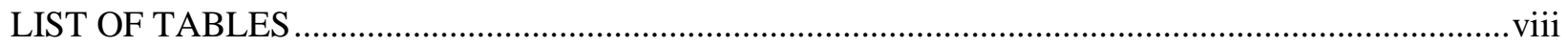

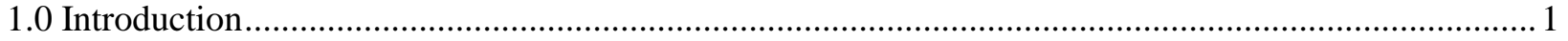

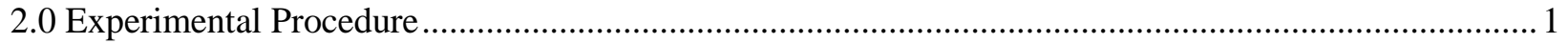

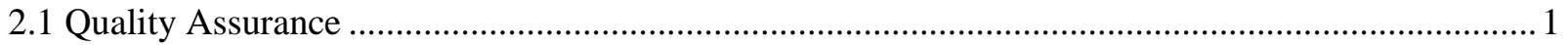

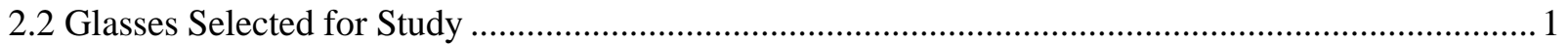

2.3 Glass Composition Analysis ..................................................................................................

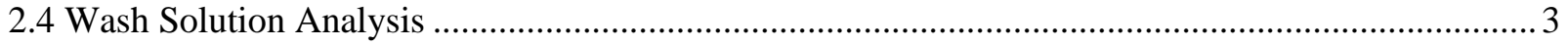

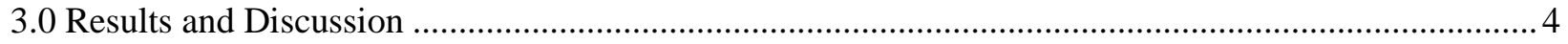

3.1 Review of the Glass Composition Measurements .......................................................................... 4

3.2 Review and Evaluation of Wash Solution Measurements............................................................... 5

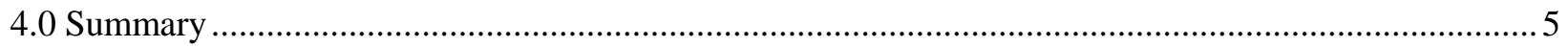

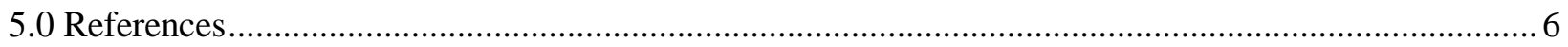

Appendix A Tables Supporting the Chemical Analysis of the Study Glasses ...................................A-1

Appendix B Tables and Exhibits Supporting the Wash Solution Chemical Composition Analysis ... B-1 


\section{LIST OF TABLES}

Table 2-1. Glass Identifiers Included in This Study ............................................................................ 2

Table 2-2. Preparation and Measurement Methods Used in Reporting the Concentrations of Each of the Analytes of the Study Glasses ........................................................................................................ 3

Table 2-3. Measurement Methods Used in Reporting the Concentrations of Each of the Analytes of the

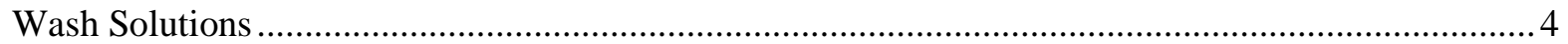




\section{LIST OF ABBREVIATIONS}

$\begin{array}{ll}\text { DOE } & \text { U.S. Department of Energy } \\ \text { IC } & \text { Ion Chromatography } \\ \text { ICP-AES } & \text { Inductively Coupled Plasma - Atomic Emission Spectroscopy } \\ \text { ICP-MS } & \text { Inductively Coupled Plasma - Mass Spectroscopy } \\ \text { HLW } & \text { High-Level Waste } \\ \text { KH } & \text { Potassium hydroxide digestion } \\ \text { LAW } & \text { Low-Activity Waste } \\ \text { LM } & \text { Lithium Metaborate fusion } \\ \text { LRM } & \text { Low-level Reference Material } \\ \text { ORP } & \text { Office of River Protection } \\ \text { PF } & \text { Peroxide Fusion } \\ \text { PNNL } & \text { Pacific Northwest National Laboratory } \\ \text { PPm } & \text { parts per million } \\ \text { SRNL } & \text { Savannah River National Laboratory } \\ \text { SSM } & \text { Sulfur Saturated Melt } \\ \text { TTQAP } & \text { Task Technical and Quality Assurance Plan } \\ \text { wt \% } & \text { weight percent } \\ \text { WTP } & \text { Hanford Tank Waste Treatment and Immobilization Plant }\end{array}$




\subsection{Introduction}

The U.S. Department of Energy (DOE) Office of River Protection (ORP) has requested that the Savannah River National Laboratory (SRNL) provide expert evaluation and experimental work in support of the River Protection Project vitrification technology development program. DOE is building the Hanford Tank Waste Treatment and Immobilization Plant (WTP) at the Hanford Site in Washington to remediate 55 million gallons of radioactive waste that is temporarily stored in 177 underground tanks. The low-activity waste (LAW) fraction will be partitioned from the high-level waste (HLW). Both the LAW and HLW will then be vitrified into borosilicate glass using Joule-heated ceramic melters.

Efforts are being made to increase the loading of Hanford tank wastes in the glass while conforming to processing requirements and product quality regulations. DOE-ORP has requested that SRNL support the advancement of glass formulations and process control strategies in key technical areas, as defined in the Task Technical and Quality Assurance Plan (TTQAP). ${ }^{1}$ Two of these areas are enhancing waste glass property/composition models and broadening the compositional regions over which those models are applicable.

In this report, SRNL provides chemical analysis for several simulated LAW glass compositions, as well as chemical analysis of the wash solutions resulting from the preparation of these glasses. The Pacific Northwest National Laboratory (PNNL) selected and fabricated these glasses as part of a study on sulfur retention. The resulting data will be used in the development of improved property/composition models for LAW glass production at Hanford.

\subsection{Experimental Procedure}

\subsection{Quality Assurance}

Requirements for performing reviews of technical reports and the extent of review are established in Savannah River Site Manual E7, Procedure 2.60. SRNL documents the extent and type of review using the SRNL Technical Report Design Checklist contained in WSRC-IM-2002-00011, Rev. 2. Laboratory data for this study were recorded in the SRNL Electronic Laboratory Notebook system, experiment C348900079-20.

\subsection{Glasses Selected for Study}

The glass compositions in this study were selected and fabricated at PNNL. Identifiers for each of the 16 glasses are listed in Table 2-1. The suffixes 950 through 1200 indicate the melting temperature $\left({ }^{\circ} \mathrm{C}\right)$ used in producing the glass samples. The SSM suffix indicates that these glasses were fabricated from sulfur saturated melts. In the sections that follow, the methods used for measuring chemical compositions of the glasses and wash solutions are described, and brief reviews of the resulting data are provided. Detailed data from these analyses are included in the appendices. 
SRNL-STI-2018-00071

Revision 0

Table 2-1. Glass Identifiers Included in This Study

\begin{tabular}{||c||}
\hline Glass ID \\
\hline LAWA161-950-SSM \\
\hline LAWA161-1000-SSM \\
\hline LAWA161-1100-SSM \\
\hline LAWA161-1200-SSM \\
\hline ORPLG27-950-SSM \\
\hline ORPLG27-1000-SSM \\
\hline ORPLG27-1100-SSM \\
\hline ORPLG27-1200-SSM \\
\hline LAWB99-950-SSM \\
\hline LAWB99-1000-SSM \\
\hline LAWB99-1100-SSM \\
\hline LAWB99-1200-SSM \\
\hline 161SAS-950-SSM \\
\hline 161SAS-1000-SSM \\
\hline 161SAS-1100-SSM \\
\hline 161SAS-1200-SSM \\
\hline
\end{tabular}

\subsection{Glass Composition Analysis}

Chemical analyses were performed on a representative sample of each of the glasses listed in Table 2-1 to allow for comparisons with the targeted compositions. Three dissolution techniques, sodium peroxide fusion (PF), ${ }^{2}$ lithium metaborate fusion (LM), ${ }^{3}$ and potassium hydroxide fusion (KH), ${ }^{4}$ were used for preparing each of the glass samples for analysis.

Each of the prepared samples was analyzed twice for each element of interest by Inductively Coupled Plasma - Atomic Emission Spectroscopy (ICP-AES) ${ }^{5}$ or ion chromatography (IC). ${ }^{6}$ A sample of the lowlevel reference material (LRM) ${ }^{7}$ glass was included as a check of the performance of the ICP-AES and IC instruments. The LRM composition reported as the "Consensus Average" is used as the reference composition of this glass for the purposes of this study. ${ }^{7}$ The prepared samples from the LAWA161-series glasses were each measured a single time by Inductively Coupled Plasma - Mass Spectroscopy (ICP-MS) ${ }^{8}$ to determine cesium concentrations. The preparation and measurement methods used for each of the reported glass components are listed in Table 2-2. 
Table 2-2. Preparation and Measurement Methods Used in Reporting the Concentrations of Each of the Analytes of the Study Glasses

\begin{tabular}{|c|c|c||}
\hline Analyte & $\begin{array}{c}\text { Preparation } \\
\text { Method }\end{array}$ & $\begin{array}{c}\text { Measurement } \\
\text { Method }\end{array}$ \\
\hline $\mathrm{Al}$ & $\mathrm{LM}$ & ICP-AES \\
\hline $\mathrm{B}$ & $\mathrm{PF}$ & ICP-AES \\
\hline $\mathrm{Ca}$ & $\mathrm{LM}$ & ICP-AES \\
\hline $\mathrm{Cl}$ & $\mathrm{KH}$ & IC \\
\hline $\mathrm{Cr}$ & $\mathrm{LM}$ & ICP-AES \\
\hline $\mathrm{Cs}$ & $\mathrm{LM}$ & ICP-MS \\
\hline $\mathrm{F}$ & $\mathrm{KH}$ & $\mathrm{IC}$ \\
\hline $\mathrm{Fe}$ & $\mathrm{LM}$ & ICP-AES \\
\hline $\mathrm{K}$ & $\mathrm{LM}$ & ICP-AES \\
\hline $\mathrm{Li}$ & $\mathrm{PF}$ & ICP-AES \\
\hline $\mathrm{Mg}$ & $\mathrm{LM}$ & ICP-AES \\
\hline $\mathrm{Mn}$ & $\mathrm{LM}$ & ICP-AES \\
\hline $\mathrm{Na}$ & $\mathrm{LM}$ & ICP-AES \\
\hline $\mathrm{Ni}$ & $\mathrm{LM}$ & ICP-AES \\
\hline $\mathrm{P}$ & $\mathrm{LM}$ & ICP-AES \\
\hline $\mathrm{Pb}$ & $\mathrm{LM}$ & ICP-AES \\
\hline $\mathrm{S}$ & $\mathrm{LM}$ & ICP-AES \\
\hline $\mathrm{Si}$ & $\mathrm{PF}$ & ICP-AES \\
\hline $\mathrm{Sn}$ & $\mathrm{LM}$ & ICP-AES \\
\hline $\mathrm{V}$ & $\mathrm{LM}$ & ICP-AES \\
\hline $\mathrm{Zn}$ & $\mathrm{LM}$ & ICP-AES \\
\hline $\mathrm{Zr}$ & $\mathrm{LM}$ & ICP-AES \\
\hline
\end{tabular}

\subsection{Wash Solution Analysis}

Chemical analyses were performed on a representative sample of each of the wash solutions from the SSM glasses listed in Table 2-1. These wash solutions were prepared at PNNL and provided to SRNL for analysis. Each of the samples was analyzed in duplicate for each element of interest by ICP-AES ${ }^{5}$ and IC. ${ }^{6}$ The samples from the LAWA161-series glasses were each measured a single time by ICP-MS to determine cesium concentrations. The measurement methods used for each of the reported wash solution components are listed in Table 2-3.

Note that PNNL provided a second sample of the wash solution resulting from the fabrication of glass LAWA161-950-SSM for analysis. The suffix -W2 was added to identify this sample. Cesium concentration was not determined for this second sample. 
SRNL-STI-2018-00071

Revision 0

Table 2-3. Measurement Methods Used in Reporting the Concentrations of Each of the Analytes of the Wash Solutions

\begin{tabular}{|c|c|}
\hline Analyte & $\begin{array}{c}\text { Measurement } \\
\text { Method }\end{array}$ \\
\hline $\mathrm{Al}$ & ICP-AES \\
\hline $\mathrm{B}$ & ICP-AES \\
\hline $\mathrm{Ca}$ & ICP-AES \\
\hline $\mathrm{Cl}^{-}$ & IC \\
\hline $\mathrm{Cr}$ & ICP-AES \\
\hline $\mathrm{Cs}$ & ICP-MS \\
\hline $\mathrm{F}-$ & IC \\
\hline $\mathrm{Fe}$ & ICP-AES \\
\hline $\mathrm{K}$ & ICP-AES \\
\hline $\mathrm{Li}$ & ICP-AES \\
\hline $\mathrm{Mg}$ & ICP-AES \\
\hline $\mathrm{Na}$ & ICP-AES \\
\hline $\mathrm{Ni}$ & ICP-AES \\
\hline $\mathrm{P}$ & ICP-AES \\
\hline $\mathrm{Pb}$ & ICP-AES \\
\hline $\mathrm{S}$ & ICP-AES \\
\hline $\mathrm{Si}$ & ICP-AES \\
\hline $\mathrm{Sn}$ & ICP-AES \\
\hline $\mathrm{Ti}$ & ICP-AES \\
\hline $\mathrm{V}$ & ICP-AES \\
\hline $\mathrm{Zn}$ & ICP-AES \\
\hline $\mathrm{Zr}$ & ICP-AES \\
\hline
\end{tabular}

\subsection{Results and Discussion}

\subsection{Review of the Glass Composition Measurements}

Table A-1 and Table A-2 in Appendix A provide the elemental concentration measurements in wt \% for the study glasses. The duplicate measurements for each prepared glass sample are shown. Elemental measurements for the LRM glass are also included in these tables. The LRM measurements are within the acceptability limits utilized by SRNL. ${ }^{5}$ Table A-3 in Appendix A provides the elemental cesium concentrations measured in the prepared glass samples and the dilution factors used to convert the values to wt \% oxide. The data in these tables are provided so that the values are readily available should they be of interest for future reviews.

The duplicate (except for cesium) measurements for each analyte were converted to oxides using the appropriate gravimetric factors and averaged to determine a representative chemical composition for each glass. A sum of oxides was also computed for each glass using the averaged, measured values. All the measured sums of oxides for the study glasses fall within the interval of 96.2 to $100.4 \mathrm{wt} \%$, indicating acceptable recovery of the glass components. Table A-4 in Appendix A provides a summary of the average compositions as well as the targeted compositions and some relative differences. Entries in Table A-4 show the relative differences between the measured values and the targeted values for those oxides with measured values that were above the limits of detection. 


\subsection{Review and Evaluation of Wash Solution Measurements}

Table B-1 and Table B-2 in Appendix B provide the elemental concentration measurements in mg/L for the wash solutions as measured by ICP-AES. Table B-3 in Appendix B provides the anion concentration measurements in mg/L for the wash solutions as measured by IC. Table B-4 in Appendix B provides the cesium concentration measurements in $\mathrm{mg} / \mathrm{L}$ for the wash solutions as measured by ICP-MS. The data in these tables are provided so that the values are readily available should they be of interest for future reviews.

The duplicate measurements for each analyte (except for cesium) for each wash solution were averaged to determine a representative chemical composition for each solution. Table B-5 in Appendix B provides a summary of the average measured compositions of the wash solutions.

Note that no significant differences were observed among the measured concentration values for samples LAWA161-950-SSM-W and LAWA161-950-SSM-W2.

\subsection{Summary}

In this report, SRNL provides chemical analysis for several simulated LAW glass compositions, as well as chemical analysis of the wash solutions resulting from the preparation of these glasses. PNNL selected and fabricated these glasses as part of a study on sulfur retention in glasses as a function of melting temperature.

Chemical analyses were performed on a representative sample of each of the study glasses to allow for comparisons with the targeted compositions. Three dissolution techniques, sodium peroxide fusion, lithium metaborate fusion, and potassium hydroxide fusion, were used for preparing each of the glass samples for analysis. Each of the samples was analyzed twice for each element of interest by ICP-AES or IC. Cesium concentrations were measured a single time by ICP-MS. A glass standard was also measured as a check of the performance of the analytical instruments. Average concentrations of each of the glass components were determined and reported.

Chemical analyses were also performed on a representative sample of each of the wash solutions resulting from the preparation of the SSM versions of the study glasses. The samples were analyzed in duplicate for each element of interest by ICP-AES or IC. Cesium concentrations were measured by ICP-MS. Average concentrations of each analyte were determined and reported.

These results will be used by PNNL in the development of improved property/composition models for LAW glass production at Hanford. 


\subsection{References}

1. Fox, K. M., "Task Technical and Quality Assurance Plan for Hanford Waste Glass Development and Characterization," U.S. Department of Energy Report SRNL-RP-2013-00692, Revision 1, Savannah River National Laboratory, Aiken, SC (2016).

2. Best, D. R., "Dissolution of Glass, Sludge, and Slurry Samples Using $\mathrm{Na}_{2} \mathrm{O}_{2} / \mathrm{NaOH} / \mathrm{HCl}$," Manual L29, ITS-0040, Revision 2, Savannah River National Laboratory, Aiken, SC (2013).

3. Best, D. R., "Lithium Metaborate Fusion Preparation,” Manual L29, ITS-0071, Revision 3, Savannah River National Laboratory, Aiken, SC (2015).

4. “Sample Dissolution Using Potassium Hydroxide Fusion,” Manual L29, ITS-0035, Revision 3, Savannah River National Laboratory, Aiken, SC (2015).

5. Best, D. R., “Inductively Coupled Plasma-Atomic Emission Spectrometer, Agilent 730 ES,” Manual L29, Procedure ITS-0079, Revision 5, Savannah River National Laboratory, Aiken, SC (2014).

6. Best, D. R., “Anion Analysis Using the Dionex DX-500 and ICS-5000 Ion Chromatograph,” Manual L29, Procedure ITS-0027, Revision 3, Savannah River National Laboratory, Aiken, SC (2011).

7. Ebert, W. L. and S. F. Wolfe, "Round-robin Testing of a Reference Glass for Low-Activity Waste Forms," U.S. Department of Energy Report ANL-99/22, Argonne National Laboratory, Argonne, IL (1999).

8. "Inductively Coupled Plasma - Mass Spectrometer Elemental and Isotopic Analysis for Aqueous Liquid Samples Agilent 7700x,” Manual L16.1, Procedure ADS-1578, Revision 3, Savannah River National Laboratory, Aiken, SC (2017).

9. JMP ${ }^{\mathrm{TM}}$ Pro, Ver. 11.2.1, [Computer Software] SAS Institute Inc., Cary, NC (2014). 
SRNL-STI-2018-00071

Revision 0

Appendix A Tables Supporting the Chemical Analysis of the Study Glasses 
Table A-1. ICP-AES and IC Measurement Data (elemental wt \%) for the Study Glasses (Part 1 of 2)

\begin{tabular}{|c|c|c|c|c|c|c|c|c|c|c|c|}
\hline Sample ID & Lab ID & $\overline{\mathrm{Al}}$ & $\overline{\mathbf{B}}$ & 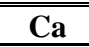 & $\mathrm{CCl}$ & $\mathrm{Cr}$ & $\bar{F}$ & Fe & $\mathbf{K}$ & $\overline{\mathbf{L i}}$ & Mg \\
\hline LRM & - & 5.03 & 2.45 & 0.386 & $<0.0500$ & 0.124 & 0.886 & 1.10 & 1.19 & $<0.100$ & $<0.100$ \\
\hline LAWA161-950-SSM-S & S-7491 & 4.93 & 4.07 & 5.53 & 0.330 & $<0.100$ & $<0.0500$ & 0.751 & 0.291 & $<0.100$ & 0.582 \\
\hline LAWA161-950-SSM-S & S-7491 & 4.91 & 3.80 & 5.45 & 0.339 & $<0.100$ & $<0.0500$ & 0.752 & 0.293 & $<0.100$ & 0.580 \\
\hline LAWA161-1000-SSM-S & S-7492 & 5.48 & 3.87 & 6.04 & 0.355 & $<0.100$ & $<0.0500$ & 0.755 & 0.324 & $<0.100$ & 0.649 \\
\hline LAWA161-1000-SSM-S & S-7492 & 5.45 & 4.34 & 5.92 & 0.342 & $<0.100$ & $<0.0500$ & 0.756 & 0.320 & $<0.100$ & 0.646 \\
\hline LAWA161-1100-SSM-S & S-7493 & 5.44 & 4.24 & 5.98 & 0.328 & $<0.100$ & $<0.0500$ & 0.726 & 0.321 & $<0.100$ & 0.643 \\
\hline LAWA161-1100-SSM-S & S-7493 & 5.40 & 4.11 & 5.97 & 0.377 & $<0.100$ & $<0.0500$ & 0.712 & 0.311 & $<0.100$ & 0.628 \\
\hline LAWA161-1200-SSM-S & S-7494 & 5.46 & 4.18 & 5.96 & 0.391 & $<0.100$ & $<0.0500$ & 0.698 & 0.326 & $<0.100$ & 0.618 \\
\hline LAWA161-1200-SSM-S & S-7494 & 5.29 & 4.00 & 5.82 & 0.332 & $<0.100$ & $<0.0500$ & 0.687 & 0.327 & $<0.100$ & 0.616 \\
\hline ORPLG27-950-SSM-S & S-7495 & 3.12 & 2.23 & 1.97 & $<0.0500$ & 0.214 & $<0.0500$ & 0.225 & 3.77 & $<0.100$ & 0.265 \\
\hline ORPLG27-950-SSM-S & S-7495 & 2.99 & 2.17 & 1.95 & $<0.0500$ & 0.211 & $<0.0500$ & 0.225 & 3.63 & $<0.100$ & 0.262 \\
\hline ORPLG27-1000-SSM-S & S-7496 & 2.99 & 2.39 & 1.94 & $<0.0500$ & 0.221 & 0.0554 & 0.217 & 3.68 & $<0.100$ & 0.253 \\
\hline ORPLG27-1000-SSM-S & S-7496 & 3.27 & 2.41 & 2.12 & $<0.0500$ & 0.243 & 0.0550 & 0.215 & 4.02 & $<0.100$ & 0.283 \\
\hline ORPLG27-1100-SSM-S & S-7497 & 3.25 & 2.33 & 2.13 & $<0.0500$ & 0.272 & $<0.0500$ & 0.221 & 4.02 & $<0.100$ & 0.278 \\
\hline ORPLG27-1100-SSM-S & S-7497 & 3.34 & 2.29 & 2.12 & $<0.0500$ & 0.270 & $<0.0500$ & 0.219 & 4.18 & $<0.100$ & 0.277 \\
\hline ORPLG27-1200-SSM-S & S-7498 & 3.28 & 2.16 & 2.10 & $<0.0500$ & 0.282 & 0.0590 & 0.219 & 3.96 & $<0.100$ & 0.278 \\
\hline ORPLG27-1200-SSM-S & S-7498 & 3.32 & 2.40 & 2.10 & $<0.0500$ & 0.277 & 0.0633 & 0.214 & 4.08 & $<0.100$ & 0.274 \\
\hline LAWB99-950-SSM-S & S-7499 & 4.96 & 3.37 & 6.83 & $<0.0500$ & $<0.100$ & $<0.0500$ & 0.873 & 0.314 & 1.32 & 0.654 \\
\hline LAWB99-950-SSM-S & S-7499 & 4.92 & 3.16 & 6.75 & $<0.0500$ & $<0.100$ & $<0.0500$ & 0.874 & 0.303 & 1.28 & 0.646 \\
\hline LAWB99-1000-SSM-S & S-7500 & 4.89 & 3.05 & 6.74 & $<0.0500$ & $<0.100$ & $<0.0500$ & 0.851 & 0.296 & 1.27 & 0.639 \\
\hline LAWB99-1000-SSM-S & S-7500 & 5.39 & 2.97 & 7.20 & $<0.0500$ & $<0.100$ & $<0.0500$ & 0.853 & 0.338 & 1.25 & 0.698 \\
\hline LAWB99-1100-SSM-S & S-7501 & 5.27 & 3.33 & 7.15 & $<0.0500$ & $<0.100$ & $<0.0500$ & 0.836 & 0.328 & 1.35 & 0.693 \\
\hline LAWB99-1100-SSM-S & S-7501 & 5.21 & 3.36 & 7.16 & $<0.0500$ & $<0.100$ & $<0.0500$ & 0.832 & 0.331 & 1.35 & 0.695 \\
\hline LAWB99-1200-SSM-S & S-7502 & 5.25 & 3.28 & 7.20 & $<0.0500$ & $<0.100$ & $<0.0500$ & 0.818 & 0.324 & 1.33 & 0.683 \\
\hline LAWB99-1200-SSM-S & S-7502 & 5.19 & 3.22 & 7.09 & $<0.0500$ & $<0.100$ & $<0.0500$ & 0.803 & 0.324 & 1.31 & 0.674 \\
\hline 161SAS-950-SSM-S & S-7503 & 5.15 & 4.30 & 5.72 & $<0.0500$ & $<0.100$ & $<0.0500$ & 0.727 & $<0.100$ & $<0.100$ & 0.603 \\
\hline 161SAS-950-SSM-S & S-7503 & 5.02 & 4.23 & 5.58 & $<0.0500$ & $<0.100$ & $<0.0500$ & 0.797 & $<0.100$ & $<0.100$ & 0.593 \\
\hline 161SAS-1000-SSM-S & S-7504 & 4.94 & 4.23 & 5.48 & $<0.0500$ & $<0.100$ & $<0.0500$ & 0.782 & $<0.100$ & $<0.100$ & 0.583 \\
\hline 161SAS-1000-SSM-S & S-7504 & 4.85 & 3.92 & 5.49 & $<0.0500$ & $<0.100$ & $<0.0500$ & 0.776 & $<0.100$ & $<0.100$ & 0.578 \\
\hline 161SAS-1100-SSM-S & S-7505 & 5.34 & 3.84 & 5.92 & $<0.0500$ & $<0.100$ & $<0.0500$ & 0.749 & $<0.100$ & $<0.100$ & 0.630 \\
\hline 161SAS-1100-SSM-S & S-7505 & 5.29 & 4.39 & 5.93 & $<0.0500$ & $<0.100$ & $<0.0500$ & 0.746 & $<0.100$ & $<0.100$ & 0.621 \\
\hline 161SAS-1200-SSM-S & S-7506 & 5.40 & 4.31 & 5.98 & $<0.0500$ & $<0.100$ & $<0.0500$ & 0.740 & $<0.100$ & $<0.100$ & 0.630 \\
\hline 161SAS-1200-SSM-S & S-7506 & 5.37 & 4.11 & 5.88 & $<0.0500$ & $<0.100$ & $<0.0500$ & 0.747 & $<0.100$ & $<0.100$ & 0.616 \\
\hline
\end{tabular}


Table A-2. ICP-AES and IC Measurement Data (elemental wt \%) for the Study Glasses (Part 2 of 2)

\begin{tabular}{|c|c|c|c|c|c|c|c|c|c|c|c|c|}
\hline Sample ID & Lab ID & $\mathrm{Na}$ & $\mathrm{Ni}$ & $\overline{\mathbf{P}}$ & $\mathbf{P b}$ & $\mathrm{S}$ & $\overline{\mathrm{Si}}$ & Sn & $\overline{T i}$ & $\mathbf{V}$ & $\overline{Z n}$ & $\overline{Z r}$ \\
\hline LRM & - & 15.4 & 0.114 & 0.201 & $<0.100$ & 0.0856 & 24.0 & $<0.100$ & $<0.100$ & $<0.100$ & $<0.100$ & 0.678 \\
\hline LAWA161-950-SSM-S & S-7491 & 14.4 & $<0.100$ & $<0.100$ & $<0.100$ & 0.444 & 17.8 & $<0.100$ & $<0.100$ & 0.508 & 2.26 & 1.99 \\
\hline LAWA161-950-SSM-S & S-7491 & 14.3 & $<0.100$ & $<0.100$ & $<0.100$ & 0.455 & 16.9 & $<0.100$ & $<0.100$ & 0.505 & 2.22 & 1.97 \\
\hline LAWA161-1000-SSM-S & S-7492 & 16.0 & $<0.100$ & $<0.100$ & $<0.100$ & 0.501 & 16.0 & $<0.100$ & $<0.100$ & 0.576 & 2.50 & 2.21 \\
\hline LAWA161-1000-SSM-S & S-7492 & 15.8 & $<0.100$ & $<0.100$ & $<0.100$ & 0.500 & 15.8 & $<0.100$ & $<0.100$ & 0.568 & 2.49 & 2.19 \\
\hline LAWA161-1100-SSM-S & S-7493 & 16.0 & $<0.100$ & $<0.100$ & $<0.100$ & 0.526 & 15.4 & $<0.100$ & $<0.100$ & 0.565 & 2.49 & 2.16 \\
\hline LAWA161-1100-SSM-S & S-7493 & 16.0 & $<0.100$ & $<0.100$ & $<0.100$ & 0.522 & 14.9 & $<0.100$ & $<0.100$ & 0.549 & 2.49 & 2.16 \\
\hline LAWA161-1200-SSM-S & S-7494 & 16.0 & $<0.100$ & $<0.100$ & $<0.100$ & 0.528 & 16.5 & $<0.100$ & $<0.100$ & 0.545 & 2.50 & 2.21 \\
\hline LAWA161-1200-SSM-S & S-7494 & 15.5 & $<0.100$ & $<0.100$ & $<0.100$ & 0.526 & 15.9 & $<0.100$ & $<0.100$ & 0.545 & 2.41 & 2.14 \\
\hline ORPLG27-950-SSM-S & S-7495 & 15.3 & $<0.100$ & $<0.100$ & $<0.100$ & 0.398 & 19.0 & 2.34 & $<0.100$ & $<0.100$ & 2.06 & 4.41 \\
\hline ORPLG27-950-SSM-S & S-7495 & 15.3 & $<0.100$ & $<0.100$ & $<0.100$ & 0.383 & 19.2 & 2.36 & $<0.100$ & $<0.100$ & 2.05 & 4.37 \\
\hline ORPLG27-1000-SSM-S & S-7496 & 15.1 & $<0.100$ & $<0.100$ & $<0.100$ & 0.388 & 18.6 & 2.33 & $<0.100$ & $<0.100$ & 2.04 & 4.34 \\
\hline ORPLG27-1000-SSM-S & S-7496 & 16.3 & $<0.100$ & $<0.100$ & $<0.100$ & 0.436 & 19.3 & 2.59 & $<0.100$ & $<0.100$ & 2.23 & 4.74 \\
\hline ORPLG27-1100-SSM-S & S-7497 & 16.4 & $<0.100$ & $<0.100$ & $<0.100$ & 0.429 & 18.0 & 2.61 & $<0.100$ & $<0.100$ & 2.25 & 4.78 \\
\hline ORPLG27-1100-SSM-S & S-7497 & 16.4 & $<0.100$ & $<0.100$ & $<0.100$ & 0.427 & 18.1 & 2.62 & $<0.100$ & $<0.100$ & 2.26 & 4.82 \\
\hline ORPLG27-1200-SSM-S & S-7498 & 16.3 & $<0.100$ & $<0.100$ & $<0.100$ & 0.479 & 18.9 & 2.57 & $<0.100$ & $<0.100$ & 2.21 & 4.74 \\
\hline ORPLG27-1200-SSM-S & S-7498 & 16.3 & $<0.100$ & $<0.100$ & $<0.100$ & 0.481 & 19.5 & 2.60 & $<0.100$ & $<0.100$ & 2.22 & 4.75 \\
\hline LAWB99-950-SSM-S & S-7499 & 7.77 & $<0.100$ & $<0.100$ & $<0.100$ & 0.594 & 19.8 & $<0.100$ & $<0.100$ & 0.632 & 2.71 & 2.37 \\
\hline LAWB99-950-SSM-S & S-7499 & 7.62 & $<0.100$ & $<0.100$ & $<0.100$ & 0.590 & 19.6 & $<0.100$ & $<0.100$ & 0.616 & 2.66 & 2.34 \\
\hline LAWB99-1000-SSM-S & S-7500 & 7.66 & $<0.100$ & $<0.100$ & $<0.100$ & 0.605 & 18.9 & $<0.100$ & $<0.100$ & 0.609 & 2.65 & 2.32 \\
\hline LAWB99-1000-SSM-S & S-7500 & 8.31 & $<0.100$ & $<0.100$ & $<0.100$ & 0.668 & 18.8 & $<0.100$ & $<0.100$ & 0.672 & 2.91 & 2.55 \\
\hline LAWB99-1100-SSM-S & S-7501 & 8.26 & $<0.100$ & $<0.100$ & $<0.100$ & 0.700 & 18.6 & $<0.100$ & $<0.100$ & 0.666 & 2.87 & 2.51 \\
\hline LAWB99-1100-SSM-S & S-7501 & 8.20 & $<0.100$ & $<0.100$ & $<0.100$ & 0.724 & 17.8 & $<0.100$ & $<0.100$ & 0.666 & 2.87 & 2.51 \\
\hline LAWB99-1200-SSM-S & S-7502 & 8.54 & $<0.100$ & $<0.100$ & $<0.100$ & 0.726 & 19.6 & $<0.100$ & $<0.100$ & 0.660 & 2.88 & 2.50 \\
\hline LAWB99-1200-SSM-S & S-7502 & 8.31 & $<0.100$ & $<0.100$ & $<0.100$ & 0.697 & 19.4 & $<0.100$ & $<0.100$ & 0.650 & 2.80 & 2.46 \\
\hline 161SAS-950-SSM-S & S-7503 & 15.9 & $<0.100$ & $<0.100$ & $<0.100$ & 0.682 & 16.4 & $<0.100$ & $<0.100$ & $<0.100$ & 2.34 & 2.09 \\
\hline 161SAS-950-SSM-S & S-7503 & 15.5 & $<0.100$ & $<0.100$ & $<0.100$ & 0.669 & 16.4 & $<0.100$ & $<0.100$ & $<0.100$ & 2.29 & 2.03 \\
\hline 161SAS-1000-SSM-S & S-7504 & 15.0 & $<0.100$ & $<0.100$ & $<0.100$ & 0.522 & 18.1 & $<0.100$ & $<0.100$ & $<0.100$ & 2.26 & 2.00 \\
\hline 161SAS-1000-SSM-S & S-7504 & 14.9 & $<0.100$ & $<0.100$ & $<0.100$ & 0.529 & 17.6 & $<0.100$ & $<0.100$ & $<0.100$ & 2.23 & 1.98 \\
\hline 161SAS-1100-SSM-S & S-7505 & 16.2 & $<0.100$ & $<0.100$ & $<0.100$ & 0.622 & 16.9 & $<0.100$ & $<0.100$ & $<0.100$ & 2.47 & 2.18 \\
\hline 161SAS-1100-SSM-S & S-7505 & 16.2 & $<0.100$ & $<0.100$ & $<0.100$ & 0.609 & 16.4 & $<0.100$ & $<0.100$ & $<0.100$ & 2.46 & 2.17 \\
\hline 161SAS-1200-SSM-S & S-7506 & 16.4 & $<0.100$ & $<0.100$ & $<0.100$ & 0.652 & 16.2 & $<0.100$ & $<0.100$ & $<0.100$ & 2.48 & 2.20 \\
\hline 161SAS-1200-SSM-S & S-7506 & 16.3 & $<0.100$ & $<0.100$ & $<0.100$ & 0.624 & 16.7 & $<0.100$ & $<0.100$ & $<0.100$ & 2.46 & 2.19 \\
\hline
\end{tabular}


Table A-3. ICP-MS Measurement Data for Cesium Concentrations in the Study Glasses

\begin{tabular}{|c|c|c|c|c||}
\hline Sample ID & LAWA161-950-SSM-S & LAWA161-1000-SSM-S & LAWA161-1100-SSM-S & LAWA161-1200-SSM-S \\
\hline Lab ID & LW9927 S-7491 & LW9928 S-7492 & LW9929 S-7493 & LW9930 S-7494 \\
\hline Measured Cs (ug/L) & $4.24 \mathrm{E}+02$ & $4.20 \mathrm{E}+02$ & $4.26 \mathrm{E}+02$ & $4.33 \mathrm{E}+02$ \\
\hline Solution Volume(L) & 0.250 & 0.250 & 0.250 & 0.250 \\
\hline Sample Mass (g) & 0.1504 & 0.1511 & 0.1512 & 0.1504 \\
\hline g Cs / g sample & $7.05 \mathrm{E}-04$ & $6.95 \mathrm{E}-04$ & $7.04 \mathrm{E}-04$ & $7.19 \mathrm{E}-04$ \\
\hline Cs2O (wt \%) & 0.07 & 0.07 & 0.07 & 0.08 \\
\hline
\end{tabular}


Table A-4. Summary of Measured Composition Data (wt \% oxide) for the Study Glasses

\begin{tabular}{|c|c|c|c|c|c|c|c|c|c|c|c|c|c|c|c|c|c|c|c|c|c|c|c|c|}
\hline \multirow{4}{*}{ LRM } & View & $\mathrm{Al}_{2} \mathbf{O}_{3}$ & $\mathbf{B}_{2} \mathbf{O}_{3}$ & $\mathrm{CaO}$ & $\mathrm{Cl}^{-}$ & $\mathrm{Cr}_{2} \mathrm{O}_{3}$ & Cs2O & $F^{-}$ & $\mathrm{Fe}_{2} \mathrm{O}_{3}$ & $\mathrm{~K}_{2} \mathrm{O}$ & $\mathrm{Li}_{2} \mathrm{O}$ & MgO & $\mathrm{Na}_{2} \mathrm{O}$ & $\mathrm{NiO}$ & $\mathbf{P}_{2} \mathrm{O}_{5}$ & PbO & $\mathrm{SO}_{3}$ & $\mathrm{SiO}_{2}$ & $\mathrm{SnO}_{2}$ & $\mathrm{TiO}_{2}$ & $\mathbf{V}_{2} \mathbf{O}_{5}$ & $\mathrm{ZnO}$ & $\mathrm{ZrO}_{2}$ & Sum \\
\hline & Measured & 9.50 & 7.87 & 0.540 & $<0.0500$ & 0.181 & & 0.886 & 1.57 & 1.43 & $<0.215$ & $<0.166$ & 20.7 & 0.146 & 0.462 & $<0.108$ & 0.214 & 51.3 & $<0.127$ & $<0.167$ & $<0.179$ & $<0.124$ & 0.916 & 96.9 \\
\hline & Targeted & 9.51 & 7.85 & 0.54 & 0 & 0.19 & & 0.86 & 1.38 & 1.48 & 0.11 & 0.1 & 20.03 & 0.19 & 0.54 & 0.1 & 0.3 & 54.2 & 0 & 0.1 & 0 & 0 & 0.93 & 98.4 \\
\hline & Percent Error & $0 \%$ & $0 \%$ & $0 \%$ & & $-5 \%$ & & $3 \%$ & $14 \%$ & $-3 \%$ & & & $3 \%$ & $-23 \%$ & $-14 \%$ & & $-29 \%$ & $-5 \%$ & & & & & $-2 \%$ & \\
\hline \multirow{3}{*}{ LAWA161-950-SSM-S } & Measured & 9.30 & 12.7 & 7.68 & 0.334 & $<0.146$ & 0.07 & $<0.0500$ & 1.07 & 0.352 & $<0.215$ & 0.963 & 19.4 & $<0.127$ & $<0.229$ & $<0.108$ & 1.12 & 37.1 & $<0.127$ & $<0.167$ & 0.904 & 2.79 & 2.68 & 97.6 \\
\hline & Targeted & 10.16 & 13.67 & 7.99 & 1.17 & 0.02 & 0.15 & 0 & 1 & 0.44 & 0 & 1 & 20.66 & 0 & 0 & 0 & 0.19 & 36.58 & 0 & 0 & 1 & 2.99 & 2.99 & 100.0 \\
\hline & Percent Error & $-8 \%$ & $-7 \%$ & $-4 \%$ & $-71 \%$ & & $-53 \%$ & & $7 \%$ & $-20 \%$ & & $-4 \%$ & $-6 \%$ & & & & $\mathrm{n} / \mathrm{a}$ & $1 \%$ & & & $-10 \%$ & $-7 \%$ & $-10 \%$ & \\
\hline \multirow{3}{*}{ LAWA161-1000-SSM-S } & Measured & 10.3 & 13.2 & 8.37 & 0.348 & $<0.146$ & 0.07 & $<0.0500$ & 1.08 & 0.388 & $<0.215$ & 1.07 & 21.4 & $<0.127$ & $<0.229$ & $<0.108$ & 1.25 & 34.0 & $<0.127$ & $<0.167$ & 1.02 & 3.10 & 2.97 & 99.7 \\
\hline & Targeted & 10.16 & 13.67 & 7.99 & 1.17 & 0.02 & 0.15 & 0 & 1 & 0.44 & 0 & 1 & 20.66 & 0 & 0 & 0 & 0.19 & 36.58 & 0 & 0 & 1 & 2.99 & 2.99 & 100.0 \\
\hline & Percent Error & $1 \%$ & $-3 \%$ & $5 \%$ & $-70 \%$ & & $-53 \%$ & & $8 \%$ & $-12 \%$ & & $7 \%$ & $4 \%$ & & & & $\mathrm{n} / \mathrm{a}$ & $-7 \%$ & & & $2 \%$ & $4 \%$ & $-1 \%$ & \\
\hline \multirow{3}{*}{ LAWA161-1100-SSM-S } & Measured & 10.2 & 13.4 & 8.36 & 0.353 & $<0.146$ & 0.07 & $<0.0500$ & 1.03 & 0.381 & $<0.215$ & 1.05 & 21.5 & $<0.127$ & $<0.229$ & $<0.108$ & 1.31 & 32.4 & $<0.127$ & $<0.167$ & 0.995 & 3.10 & 2.92 & 98.2 \\
\hline & Targeted & 10.16 & 13.67 & 7.99 & 1.17 & 0.02 & 0.15 & 0 & 1 & 0.44 & 0 & 1 & 20.66 & 0 & 0 & 0 & 0.19 & 36.58 & 0 & 0 & 1 & 2.99 & 2.99 & 100.0 \\
\hline & Percent Error & $0 \%$ & $-2 \%$ & $5 \%$ & $-70 \%$ & & $-53 \%$ & & $3 \%$ & $-13 \%$ & & $5 \%$ & $4 \%$ & & & & $\mathrm{n} / \mathrm{a}$ & $-11 \%$ & & & $-1 \%$ & $4 \%$ & $-2 \%$ & \\
\hline \multirow{3}{*}{ LAWA161-1200-SSM-S } & Measured & 10.2 & 13.2 & 8.24 & 0.361 & $<0.146$ & 0.08 & 0.0500 & 0.990 & 0.393 & $<0.215$ & 1.02 & 21.3 & $<0.127$ & 0.229 & 0.108 & 1.32 & 34.7 & 0.127 & 0.167 & 0.973 & 3.05 & 2.93 & 99.9 \\
\hline & Targeted & 10.16 & 13.67 & 7.99 & 1.17 & 0.02 & 0.15 & 0 & 1 & 0.44 & 0 & 1 & 20.66 & 0 & 0 & 0 & 0.19 & 36.58 & 0 & 0 & 1 & 2.99 & 2.99 & 100.0 \\
\hline & Percent Error & $0 \%$ & $-3 \%$ & $3 \%$ & $-69 \%$ & $0.0<$ & $-47 \%$ & 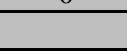 & $-1 \%$ & $-11 \%$ & 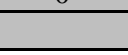 & $2 \%$ & $3 \%$ & 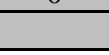 & - & - & $\mathrm{n} / \mathrm{a}$ & $-5 \%$ & - & - & $-3 \%$ & $2 \%$ & $-2 \%$ & 0.00 \\
\hline \multirow{3}{*}{ ORPLG27-950-SSM-S } & Measured & 5.78 & 7.07 & 2.74 & $<0.0500$ & 0.310 & & $<0.0500$ & 0.321 & 4.46 & $<0.215$ & 0.437 & 20.6 & $<0.127$ & $<0.229$ & $<0.108$ & 0.976 & 40.9 & 2.98 & $<0.167$ & $<0.179$ & 2.55 & 5.93 & 96.2 \\
\hline & Targeted & 6.03 & 7.92 & 2.69 & 0.23 & 0.59 & & 0.09 & 0.28 & 5.75 & 0 & 0.44 & 21 & 0.01 & 0.14 & 0.01 & 0.41 & 42.1 & 3.19 & 0 & 0 & 2.69 & 6.44 & 100.0 \\
\hline & Percent Error & $-4 \%$ & $-11 \%$ & $2 \%$ & & $-47 \%$ & & & $15 \%$ & $-22 \%$ & & $-1 \%$ & $-2 \%$ & & & & $\mathrm{n} / \mathrm{a}$ & $-3 \%$ & $-7 \%$ & & & $-5 \%$ & $-8 \%$ & \\
\hline \multirow{3}{*}{ ORPLG27-1000-SSM-S } & Measured & 5.91 & 7.72 & 2.84 & $<0.0500$ & 0.339 & & 0.0552 & 0.309 & 4.64 & $<0.215$ & 0.444 & 21.2 & $<0.127$ & $<0.229$ & $<0.108$ & 1.03 & 40.6 & 3.12 & $<0.167$ & $<0.179$ & 2.66 & 6.13 & 98.1 \\
\hline & Targeted & 6.03 & 7.92 & 2.69 & 0.23 & 0.59 & & 0.09 & 0.28 & 5.75 & 0 & 0.44 & 21 & 0.01 & 0.14 & 0.01 & 0.41 & 42. & 3.19 & 0 & 0 & 2.69 & 6.44 & 100.0 \\
\hline & Percent Error & $-2 \%$ & $-3 \%$ & $6 \%$ & & $-43 \%$ & & $-39 \%$ & $10 \%$ & $-19 \%$ & & $1 \%$ & $1 \%$ & & & & $\mathrm{n} / \mathrm{a}$ & $-4 \%$ & $-2 \%$ & & & $-1 \%$ & $-5 \%$ & \\
\hline \multirow{3}{*}{ ORPLG27-1100-SSM-S } & Measured & 6.23 & 7.43 & 2.98 & $<0.0500$ & 0.396 & & $<0.0500$ & 0.314 & 4.94 & $<0.215$ & 0.461 & 22.1 & $<0.127$ & $<0.229$ & $<0.108$ & 1.07 & 38.6 & 3.32 & $<0.167$ & $<0.179$ & 2.81 & 6.48 & 98.3 \\
\hline & Targeted & 6.03 & 7.92 & 2.69 & 0.23 & 0.59 & & 0.09 & 0.28 & 5.75 & 0 & 0.44 & 21 & 0.01 & 0.14 & 0.01 & 0.41 & 42.1 & 3.19 & 0 & 0 & 2.69 & 6.44 & 100.0 \\
\hline & Percent Error & $3 \%$ & $-6 \%$ & $11 \%$ & & $-33 \%$ & & & $12 \%$ & $-14 \%$ & & $5 \%$ & $5 \%$ & & & & $\mathrm{n} / \mathrm{a}$ & $-8 \%$ & $4 \%$ & & & $4 \%$ & $1 \%$ & \\
\hline \multirow{3}{*}{ ORPLG27-1200-SSM-S } & Measured & 6.23 & 7.33 & 2.94 & $<0.0500$ & 0.408 & & 0.0612 & 0.309 & 4.85 & $<0.215$ & 0.457 & 22.0 & $<0.127$ & $<0.229$ & $<0.108$ & 1.20 & 41.0 & 3.28 & $<0.167$ & $<0.179$ & 2.76 & 6.41 & 100.3 \\
\hline & Targeted & 6.03 & 7.92 & 2.69 & 0.23 & 0.59 & & 0.09 & 0.28 & 5.75 & 0 & 0.44 & 21 & 0.01 & 0.14 & 0.01 & 0.41 & 42 & 3.19 & 0 & 0 & 2.69 & 6.44 & 100.0 \\
\hline & Percent Error & $3 \%$ & $-7 \%$ & $9 \%$ & & $-31 \%$ & & $-32 \%$ & $10 \%$ & $-16 \%$ & & $4 \%$ & $5 \%$ & & & & $\mathrm{n} / \mathrm{a}$ & $-3 \%$ & $3 \%$ & & & $3 \%$ & $0 \%$ & \\
\hline \multirow{3}{*}{ LAWAB99-950-SSM-S } & Measured & 9.33 & 10.5 & 9.51 & $<0.0500$ & $<0.146$ & & $<0.0500$ & 1.25 & 0.372 & 2.80 & 1.08 & 10.4 & $<0.127$ & 0.229 & 0.108 & 1.48 & 42.2 & $<0.127$ & $<0.167$ & 1.11 & 3.34 & 19 & 97.6 \\
\hline & Targeted & 10.15 & 11.01 & 10.21 & 0.01 & 0.11 & & 0.07 & 1.15 & 0.41 & 3.54 & 1.15 & 10 & 0 & 0.03 & 0 & 0.75 & 43.08 & 0 & 0 & 1.24 & 3.54 & 3.54 & 100.0 \\
\hline & Percent Error & $-8 \%$ & $-5 \%$ & $-7 \%$ & r.t. & 0.11 & & & $9 \%$ & $-9 \%$ & $-21 \%$ & $-6 \%$ & $4 \%$ & 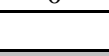 & 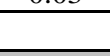 & 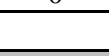 & $\mathrm{n} / \mathrm{a}$ & $-2 \%$ & 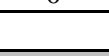 & & $-10 \%$ & $-6 \%$ & $-10 \%$ & \\
\hline \multirow{3}{*}{ LAWAB99-1000-SSM-S } & Measured & 9.71 & 9.70 & 9.75 & $<0.0500$ & $<0.146$ & & $<0.0500$ & 1.22 & 0.382 & 2.72 & 1.11 & 10.8 & $<0.127$ & $<0.229$ & $<0.108$ & 1.59 & 40.4 & $<0.127$ & $<0.167$ & 1.14 & 3.46 & 3.29 & 96.3 \\
\hline & Targeted & 10.15 & 11.01 & 10.21 & 0.01 & 0.11 & & 0.07 & 1.15 & 0.41 & 3.54 & 1.15 & 10 & 0 & 0.03 & 0 & 0.75 & 43.08 & 0 & 0 & 1.24 & 3.54 & 3.54 & 100.0 \\
\hline & Percent Error & $-4 \%$ & $-12 \%$ & $-5 \%$ & & & & & $6 \%$ & -70 & $-23 \%$ & $-3 \%$ & $8 \%$ & & & & $\mathrm{n} / \mathrm{a}$ & $-6 \%$ & & & $-8 \%$ & $-2 \%$ & $-7 \%$ & \\
\hline & Measured & 9.91 & 10.8 & 10.0 & $<0.0500$ & $<0.146$ & & $<0.0500$ & 1.19 & 0.397 & 2.90 & 1.15 & 11.1 & $<0.127$ & $<0.229$ & $<0.108$ & 1.78 & 39.0 & $<0.127$ & $<0.167$ & 1.19 & 3.57 & 3.39 & 97.4 \\
\hline LAWAB99-1100-SSM-S & Targeted & 10.15 & 11.01 & 10.21 & 0.01 & 0.11 & & 0.07 & 1.15 & 0.41 & 3.54 & 1.15 & 10 & 0 & 0.03 & 0 & 0.75 & 43.08 & 0 & 0 & 1.24 & 3.54 & 3.54 & 100.0 \\
\hline & Percent Error & $-2 \%$ & $-2 \%$ & $-2 \%$ & & & & & $3 \%$ & $-3 \%$ & $-18 \%$ & $0 \%$ & $11 \%$ & & & & $\mathrm{n} / \mathrm{a}$ & $-9 \%$ & & & $-4 \%$ & $1 \%$ & $-4 \%$ & \\
\hline & Measured & 9.86 & 10.5 & 9.99 & $<0.0500$ & $<0.146$ & & $<0.0500$ & 1.16 & 0.390 & 2.83 & 1.12 & 111.4 & $<0.127$ & $<0.229$ & $<0.108$ & 1.78 & 41.7 & $<0.127$ & $<0.167$ & 1.17 & 3.53 & 3.35 & 99.8 \\
\hline LAWAB99-1200-SSM-S & Targeted & 10.15 & 11.01 & 10.21 & 0.01 & 0.11 & & 0.07 & 1.15 & 0.41 & 3.54 & 1.15 & 10 & 0 & 0.03 & 0 & 0.75 & 43.08 & 0 & 0 & 1.24 & 3.54 & 3.54 & 100.0 \\
\hline & Percent Error & $-3 \%$ & $-5 \%$ & $-2 \%$ & & & & & $1 \%$ & $-5 \%$ & $-20 \%$ & $-3 \%$ & $14 \%$ & & & & $\mathrm{n} / \mathrm{a}$ & $-3 \%$ & & & $-6 \%$ & $0 \%$ & $-5 \%$ & \\
\hline & Measured & 9.61 & 13.7 & 7.91 & $<0.0500$ & $<0.146$ & & $<0.0500$ & 1.09 & $<0.120$ & $<0.215$ & 0.992 & 21.2 & $<0.127$ & $<0.229$ & $<0.108$ & 1.69 & 35.1 & $<0.127$ & $<0.167$ & $<0.179$ & 2.88 & 2.78 & 98.5 \\
\hline 161SAS-950-SSM-S & Targeted & 10.47 & 14.09 & 8.23 & 0 & 0 & & 0 & 1.03 & 0 & 0 & 1.03 & 21.29 & 0 & 0 & 0 & 0 & $\begin{array}{l}37.7 \\
\end{array}$ & 0 & 0 & 0 & 3.08 & 3.08 & 100.0 \\
\hline & Percent Error & $-8 \%$ & $-3 \%$ & $-4 \%$ & & & & & $6 \%$ & & & $-4 \%$ & $0 \%$ & & & & $\mathrm{n} / \mathrm{a}$ & $-7 \%$ & & & & $-6 \%$ & $-10 \%$ & \\
\hline & Measured & 9.25 & 13.1 & 7.68 & $<0.0500$ & $<0.146$ & & $<0.0500$ & 1.11 & $<0.120$ & $<0.215$ & 0.963 & 20.2 & $<0.127$ & $<0.229$ & $<0.108$ & 1.31 & 38.2 & $<0.127$ & $<0.167$ & $<0.179$ & 2.79 & 2.69 & 98.8 \\
\hline 161SAS-1000-SSM-S & Targeted & 10.47 & 14.09 & 8.23 & 0 & 0 & & 0 & 1.03 & 0 & 0 & 1.03 & 21.29 & 0 & 0 & 0 & 0 & 37. & 0 & 0 & 0 & 3.08 & 3.08 & 100.0 \\
\hline & Percent Error & $-12 \%$ & $-7 \%$ & $-7 \%$ & & & & & $8 \%$ & & & $-7 \%$ & $-5 \%$ & & & & $\mathrm{n} / \mathrm{a}$ & $1 \%$ & & & & $-9 \%$ & $-13 \%$ & \\
\hline & Measured & 10.0 & 13.2 & 8.29 & $<0.0500$ & $<0.146$ & & $<0.0500$ & 1.07 & $<0.120$ & $<0.215$ & 1.04 & 21.9 & $<0.127$ & $<0.229$ & $<0.108$ & 1.54 & 35. & $<0.127$ & $<0.167$ & $<0.179$ & 3.07 & 2.93 & 100.2 \\
\hline 161SAS-1100-SSM-S & Targeted & 10.47 & 14.09 & 8.23 & 0 & 0 & & 0 & 1.03 & 0 & 0 & 1.03 & 21.29 & 0 & 0 & 0 & 0 & 37.7 & 0 & 0 & 0 & 3.08 & 3.08 & 100.0 \\
\hline & Percent Error & $-4 \%$ & $-6 \%$ & $1 \%$ & & & & & $4 \%$ & & & $1 \%$ & $3 \%$ & & & & $\mathrm{n} / \mathrm{a}$ & $-6 \%$ & & & & $0 \%$ & $-5 \%$ & \\
\hline & Measured & 10.2 & 13.6 & 8.29 & $<0.0500$ & $<0.146$ & & $<0.0500$ & 1.06 & $<0.120$ & $<0.215$ & 1.03 & 22.0 & $<0.127$ & $<0.229$ & $<0.108$ & 1.59 & 35.1 & $<0.127$ & $<0.167$ & $<0.179$ & 3.07 & 2.96 & 100.4 \\
\hline 161SAS-1200-SSM-S & Targeted & 10.47 & 14.09 & 8.23 & 0 & 0 & & 0 & 1.03 & 0 & 0 & 1.03 & 21.29 & 0 & 0 & 0 & 0 & 37.7 & 0 & 0 & T & $\begin{array}{ll}3.08 \\
\end{array}$ & 3.08 & 100.0 \\
\hline & Percent Error & $-3 \%$ & $-3 \%$ & $1 \%$ & & & & & $3 \%$ & & & $0 \%$ & $3 \%$ & & & & $\mathrm{n} / \mathrm{a}$ & $-7 \%$ & & & & $0 \%$ & $-4 \%$ & \\
\hline
\end{tabular}


SRNL-STI-2018-00071

Revision 0

Appendix B Tables and Exhibits Supporting the Wash Solution Chemical Composition Analysis

B-1 
Table B-1. ICP-AES Measurement Data (mg/L) for the Study Glass Wash Solutions (Part 1 of 2)

\begin{tabular}{|c|c|c|c|c|c|c|c|c|c|c|c|}
\hline Sample ID & Lab ID & Al & $\mathbf{B}$ & Ca & $\mathrm{Cr}$ & Fe & $\mathbf{K}$ & $\mathbf{L i}$ & Mg & $\mathrm{Na}$ & $\mathrm{Ni}$ \\
\hline LAWA161-950-SSM-W & S-7507 & $<1.00$ & 5.41 & $<1.00$ & $<1.00$ & $<1.00$ & $<0.500$ & $<1.00$ & $<1.00$ & 44.1 & $<1.00$ \\
\hline LAWA161-950-SSM-W & S-7507 & $<1.00$ & 6.18 & $<1.00$ & $<1.00$ & $<1.00$ & $<0.500$ & $<1.00$ & $<1.00$ & 47.3 & $<1.00$ \\
\hline LAWA161-950-SSM-W2 & S-7570 & $<1.00$ & 6.13 & $<1.00$ & $<1.00$ & $<1.00$ & $<0.500$ & - & $<1.00$ & 48.1 & - \\
\hline LAWA161-950-SSM-W2 & S-7570 & $<1.00$ & 6.05 & $<1.00$ & $<1.00$ & $<1.00$ & $<0.500$ & - & $<1.00$ & 49.1 & - \\
\hline LAWA161-1000-SSM-W & S-7508 & $<1.00$ & 10.3 & 3.17 & $<1.00$ & $<1.00$ & 6.16 & $<1.00$ & $<1.00$ & 262 & $<1.00$ \\
\hline LAWA161-1000-SSM-W & S-7508 & $<1.00$ & 9.57 & 3.16 & $<1.00$ & $<1.00$ & 5.58 & $<1.00$ & $<1.00$ & 256 & $<1.00$ \\
\hline LAWA161-1100-SSM-W & S-7509 & $<1.00$ & 25.2 & 18.6 & $<1.00$ & $<1.00$ & 27.4 & $<1.00$ & $<1.00$ & 949 & $<1.00$ \\
\hline LAWA161-1100-SSM-W & S-7509 & $<1.00$ & 23.2 & 18.8 & $<1.00$ & $<1.00$ & 27.0 & $<1.00$ & $<1.00$ & 896 & $<1.00$ \\
\hline LAWA161-1200-SSM-W & S-7510 & $<1.00$ & 25.9 & 18.9 & 1.17 & $<1.00$ & 24.6 & $<1.00$ & $<1.00$ & 836 & $<1.00$ \\
\hline LAWA161-1200-SSM-W & S-7510 & $<1.00$ & 27.9 & 19.1 & 1.21 & $<1.00$ & 26.7 & $<1.00$ & $<1.00$ & 820 & $<1.00$ \\
\hline ORPLG27-950-SSM-W & S-7511 & $<1.00$ & 12.9 & $<1.00$ & 35.9 & $<1.00$ & 208 & $<1.00$ & $<1.00$ & 500 & $<1.00$ \\
\hline ORPLG27-950-SSM-W & S-7511 & $<1.00$ & 12.6 & $<1.00$ & 36.6 & $<1.00$ & 194 & $<1.00$ & $<1.00$ & 475 & $<1.00$ \\
\hline ORPLG27-1000-SSM-W & S-7512 & $<1.00$ & 17.1 & $<1.00$ & 58.5 & $<1.00$ & 282 & $<1.00$ & $<1.00$ & 676 & $<1.00$ \\
\hline ORPLG27-1000-SSM-W & S-7512 & $<1.00$ & 18.4 & $<1.00$ & 58.7 & $<1.00$ & 296 & $<1.00$ & $<1.00$ & 696 & $<1.00$ \\
\hline ORPLG27-1100-SSM-W & S-7513 & $<1.00$ & 22.2 & $<1.00$ & 53.8 & $<1.00$ & 348 & $<1.00$ & $<1.00$ & 799 & $<1.00$ \\
\hline ORPLG27-1100-SSM-W & S-7513 & $<1.00$ & 21.9 & $<1.00$ & 53.7 & $<1.00$ & 362 & $<1.00$ & $<1.00$ & 843 & $<1.00$ \\
\hline ORPLG27-1200-SSM-W & S-7514 & $<1.00$ & 29.6 & $<1.00$ & 55.5 & $<1.00$ & 375 & $<1.00$ & $<1.00$ & 911 & $<1.00$ \\
\hline ORPLG27-1200-SSM-W & S-7514 & $<1.00$ & 31.3 & $<1.00$ & 55.8 & $<1.00$ & 369 & $<1.00$ & $<1.00$ & 884 & $<1.00$ \\
\hline LAWB99-950-SSM-W & S-7515 & $<1.00$ & 6.59 & 72.6 & 5.29 & $<1.00$ & 23.0 & 45.3 & $<1.00$ & 637 & $<1.00$ \\
\hline LAWB99-950-SSM-W & S-7515 & $<1.00$ & 6.66 & 73.0 & 5.25 & $<1.00$ & 23.1 & 44.9 & $<1.00$ & 645 & $<1.00$ \\
\hline LAWB99-1000-SSM-W & S-7516 & $<1.00$ & 6.69 & 74.6 & 3.88 & $<1.00$ & 25.1 & 46.9 & $<1.00$ & 604 & $<1.00$ \\
\hline LAWB99-1000-SSM-W & S-7516 & $<1.00$ & 6.20 & 74.2 & 3.84 & $<1.00$ & 23.5 & 46.9 & $<1.00$ & 624 & $<1.00$ \\
\hline LAWB99-1100-SSM-W & S-7517 & $<1.00$ & 7.65 & 69.1 & 3.18 & $<1.00$ & 23.5 & 41.8 & $<1.00$ & 523 & $<1.00$ \\
\hline LAWB99-1100-SSM-W & S-7517 & $<1.00$ & 7.84 & 69.1 & 3.18 & $<1.00$ & 24.8 & 41.6 & $<1.00$ & 511 & $<1.00$ \\
\hline LAWB99-1200-SSM-W & S-7518 & $<1.00$ & 11.1 & 58.2 & 2.80 & $<1.00$ & 25.2 & 38.3 & $<1.00$ & 521 & $<1.00$ \\
\hline LAWB99-1200-SSM-W & S-7518 & $<1.00$ & 11.4 & 58.4 & 2.76 & $<1.00$ & 25.6 & 38.5 & $<1.00$ & 509 & $<1.00$ \\
\hline 161SAS-950-SSM-W & S-7519 & $<1.00$ & 16.6 & 7.83 & $<1.00$ & $<1.00$ & 1.12 & $<1.00$ & $<1.00$ & 617 & $<1.00$ \\
\hline 161SAS-950-SSM-W & S-7519 & $<1.00$ & 14.8 & 7.82 & $<1.00$ & $<1.00$ & 0.934 & $<1.00$ & $<1.00$ & 630 & $<1.00$ \\
\hline 161SAS-1000-SSM-W & S-7520 & $<1.00$ & 17.8 & 10.3 & $<1.00$ & $<1.00$ & 1.38 & $<1.00$ & $<1.00$ & 824 & $<1.00$ \\
\hline 161SAS-1000-SSM-W & S-7520 & $<1.00$ & 16.2 & 10.3 & $<1.00$ & $<1.00$ & 1.18 & $<1.00$ & $<1.00$ & 773 & $<1.00$ \\
\hline 161SAS-1100-SSM-W & S-7521 & $<1.00$ & 21.2 & 13.2 & $<1.00$ & $<1.00$ & 1.16 & $<1.00$ & $<1.00$ & 743 & $<1.00$ \\
\hline 161SAS-1100-SSM-W & S-7521 & $<1.00$ & 23.3 & 13.3 & $<1.00$ & $<1.00$ & 1.20 & $<1.00$ & $<1.00$ & 743 & $<1.00$ \\
\hline 161SAS-1200-SSM-W & S-7522 & $<1.00$ & 22.9 & 13.4 & $<1.00$ & $<1.00$ & 1.16 & $<1.00$ & $<1.00$ & 664 & $<1.00$ \\
\hline 161SAS-1200-SSM-W & S-7522 & $<1.00$ & 23.2 & 13.3 & $<1.00$ & $<1.00$ & 1.17 & $<1.00$ & $<1.00$ & 649 & $<1.00$ \\
\hline
\end{tabular}


Table B-2. ICP-AES Measurement Data (mg/L) for the Study Glass Wash Solutions (Part 2 of 2)

\begin{tabular}{|c|c|c|c|c|c|c|c|c|c|c|}
\hline Sample ID & Lab ID & $\mathbf{P}$ & $\mathbf{P b}$ & $\mathbf{S}$ & Si & Sn & $\mathbf{T i}$ & $\mathbf{V}$ & Zn & $\mathbf{Z r}$ \\
\hline LAWA161-950-SSM-W & S-7507 & $<2.00$ & $<1.00$ & 9.55 & 2.13 & $<1.00$ & $<1.00$ & 1.02 & $<1.00$ & $<1.00$ \\
\hline LAWA161-950-SSM-W & S-7507 & $<2.00$ & $<1.00$ & 9.47 & 2.52 & $<1.00$ & $<1.00$ & 1.03 & $<1.00$ & $<1.00$ \\
\hline LAWA161-950-SSM-W2 & S-7570 & $<2.00$ & - & 8.88 & 3.50 & - & - & $<1.00$ & $<1.00$ & $<1.00$ \\
\hline LAWA161-950-SSM-W2 & S-7570 & $<2.00$ & - & 9.35 & 3.63 & - & - & $<1.00$ & $<1.00$ & $<1.00$ \\
\hline LAWA161-1000-SSM-W & S-7508 & $<2.00$ & $<1.00$ & 165 & 2.65 & $<1.00$ & $<1.00$ & 2.48 & $<1.00$ & $<1.00$ \\
\hline LAWA161-1000-SSM-W & S-7508 & $<2.00$ & $<1.00$ & 171 & 2.21 & $<1.00$ & $<1.00$ & 2.48 & $<1.00$ & $<1.00$ \\
\hline LAWA161-1100-SSM-W & S-7509 & $<2.00$ & $<1.00$ & 570 & 2.67 & $<1.00$ & $<1.00$ & 6.25 & $<1.00$ & $<1.00$ \\
\hline LAWA161-1100-SSM-W & S-7509 & $<2.00$ & $<1.00$ & 574 & 2.39 & $<1.00$ & $<1.00$ & 6.36 & $<1.00$ & $<1.00$ \\
\hline LAWA161-1200-SSM-W & S-7510 & $<2.00$ & $<1.00$ & 515 & 1.52 & $<1.00$ & $<1.00$ & 5.96 & $<1.00$ & $<1.00$ \\
\hline LAWA161-1200-SSM-W & S-7510 & $<2.00$ & $<1.00$ & 522 & 2.00 & $<1.00$ & $<1.00$ & 5.99 & $<1.00$ & $<1.00$ \\
\hline ORPLG27-950-SSM-W & S-7511 & 2.10 & $<1.00$ & 362 & 5.57 & $<1.00$ & $<1.00$ & $<1.00$ & $<1.00$ & $<1.00$ \\
\hline ORPLG27-950-SSM-W & S-7511 & 2.06 & $<1.00$ & 361 & 5.50 & $<1.00$ & $<1.00$ & $<1.00$ & $<1.00$ & $<1.00$ \\
\hline ORPLG27-1000-SSM-W & S-7512 & 3.52 & $<1.00$ & 512 & 7.28 & $<1.00$ & $<1.00$ & $<1.00$ & $<1.00$ & $<1.00$ \\
\hline ORPLG27-1000-SSM-W & S-7512 & 3.77 & $<1.00$ & 509 & 8.00 & $<1.00$ & $<1.00$ & $<1.00$ & $<1.00$ & $<1.00$ \\
\hline ORPLG27-1100-SSM-W & S-7513 & 3.85 & $<1.00$ & 605 & 3.03 & $<1.00$ & $<1.00$ & $<1.00$ & $<1.00$ & $<1.00$ \\
\hline ORPLG27-1100-SSM-W & S-7513 & 4.06 & $<1.00$ & 602 & 2.92 & $<1.00$ & $<1.00$ & $<1.00$ & $<1.00$ & $<1.00$ \\
\hline ORPLG27-1200-SSM-W & S-7514 & 3.90 & $<1.00$ & 673 & 4.25 & $<1.00$ & $<1.00$ & $<1.00$ & $<1.00$ & $<1.00$ \\
\hline ORPLG27-1200-SSM-W & S-7514 & 4.02 & $<1.00$ & 671 & 4.74 & $<1.00$ & $<1.00$ & $<1.00$ & $<1.00$ & $<1.00$ \\
\hline LAWB99-950-SSM-W & S-7515 & $<2.00$ & $<1.00$ & 615 & $<1.00$ & $<1.00$ & $<1.00$ & 5.42 & $<1.00$ & $<1.00$ \\
\hline LAWB99-950-SSM-W & S-7515 & $<2.00$ & $<1.00$ & 611 & $<1.00$ & $<1.00$ & $<1.00$ & 5.50 & $<1.00$ & $<1.00$ \\
\hline LAWB99-1000-SSM-W & S-7516 & $<2.00$ & $<1.00$ & 600 & $<1.00$ & $<1.00$ & $<1.00$ & 5.26 & $<1.00$ & $<1.00$ \\
\hline LAWB99-1000-SSM-W & S-7516 & $<2.00$ & $<1.00$ & 586 & $<1.00$ & $<1.00$ & $<1.00$ & 5.24 & $<1.00$ & $<1.00$ \\
\hline LAWB99-1100-SSM-W & S-7517 & $<2.00$ & $<1.00$ & 524 & $<1.00$ & $<1.00$ & $<1.00$ & 5.08 & $<1.00$ & $<1.00$ \\
\hline LAWB99-1100-SSM-W & S-7517 & $<2.00$ & $<1.00$ & 530 & $<1.00$ & $<1.00$ & $<1.00$ & 5.06 & $<1.00$ & $<1.00$ \\
\hline LAWB99-1200-SSM-W & S-7518 & $<2.00$ & $<1.00$ & 492 & $<1.00$ & $<1.00$ & $<1.00$ & 5.03 & $<1.00$ & $<1.00$ \\
\hline LAWB99-1200-SSM-W & S-7518 & $<2.00$ & $<1.00$ & 477 & $<1.00$ & $<1.00$ & $<1.00$ & 5.02 & $<1.00$ & $<1.00$ \\
\hline 161SAS-950-SSM-W & S-7519 & $<2.00$ & $<1.00$ & 430 & 3.44 & $<1.00$ & $<1.00$ & $<1.00$ & $<1.00$ & $<1.00$ \\
\hline 161SAS-950-SSM-W & S-7519 & $<2.00$ & $<1.00$ & 431 & 2.77 & $<1.00$ & $<1.00$ & $<1.00$ & $<1.00$ & $<1.00$ \\
\hline 161SAS-1000-SSM-W & S-7520 & $<2.00$ & $<1.00$ & 503 & 5.45 & $<1.00$ & $<1.00$ & $<1.00$ & $<1.00$ & $<1.00$ \\
\hline 161SAS-1000-SSM-W & S-7520 & $<2.00$ & $<1.00$ & 501 & 5.02 & $<1.00$ & $<1.00$ & $<1.00$ & $<1.00$ & $<1.00$ \\
\hline 161SAS-1100-SSM-W & S-7521 & $<2.00$ & $<1.00$ & 484 & 2.65 & $<1.00$ & $<1.00$ & $<1.00$ & $<1.00$ & $<1.00$ \\
\hline 161SAS-1100-SSM-W & S-7521 & $<2.00$ & $<1.00$ & 480 & 2.71 & $<1.00$ & $<1.00$ & $<1.00$ & $<1.00$ & $<1.00$ \\
\hline 161SAS-1200-SSM-W & S-7522 & $<2.00$ & $<1.00$ & 458 & 1.33 & $<1.00$ & $<1.00$ & $<1.00$ & $<1.00$ & $<1.00$ \\
\hline 161SAS-1200-SSM-W & S-7522 & $<2.00$ & $<1.00$ & 454 & 1.47 & $<1.00$ & $<1.00$ & $<1.00$ & $<1.00$ & $<1.00$ \\
\hline
\end{tabular}


Table B-3. IC Measurement Data (mg/L) for the Study Glass Wash Solutions

\begin{tabular}{|c|c|c|c||}
\hline Sample ID & Lab ID & Cl & F \\
\hline 1ppm standard & - & 0.983 & 1.08 \\
\hline LAWA161-950-SSM-W & S-7507 & $<10.0$ & $<10.0$ \\
\hline LAWA161-950-SSM-W & S-7507 & $<10.0$ & $<10.0$ \\
\hline LAWA161-1000-SSM-W & S-7508 & 10.5 & $<10.0$ \\
\hline LAWA161-1000-SSM-W & S-7508 & 11.7 & $<10.0$ \\
\hline LAWA161-1100-SSM-W & S-7509 & 114 & $<10.0$ \\
\hline LAWA161-1100-SSM-W & S-7509 & 112 & $<10.0$ \\
\hline LAWA161-1200-SSM-W & S-7510 & 106 & $<10.0$ \\
\hline LAWA161-1200-SSM-W & S-7510 & 110 & $<10.0$ \\
\hline ORPLG27-950-SSM-W & S-7511 & 10.1 & $<10.0$ \\
\hline ORPLG27-950-SSM-W & S-7511 & 10.6 & $<10.0$ \\
\hline ORPLG27-1000-SSM-W & S-7512 & 12.2 & $<10.0$ \\
\hline ORPLG27-1000-SSM-W & S-7512 & 11.9 & $<10.0$ \\
\hline ORPLG27-1100-SSM-W & S-7513 & 17.5 & $<10.0$ \\
\hline ORPLG27-1100-SSM-W & S-7513 & 16.3 & $<10.0$ \\
\hline 1ppm standard & - & 1.00 & 1.08 \\
\hline ORPLG27-1200-SSM-W & S-7514 & 31.3 & $<10.0$ \\
\hline ORPLG27-1200-SSM-W & S-7514 & 30.4 & $<10.0$ \\
\hline LAWB99-950-SSM-W & S-7515 & $<10.0$ & $<10.0$ \\
\hline LAWB99-950-SSM-W & S-7515 & $<10.0$ & $<10.0$ \\
\hline LAWB99-1000-SSM-W & S-7516 & $<10.0$ & $<10.0$ \\
\hline LAWB99-1000-SSM-W & S-7516 & $<10.0$ & $<10.0$ \\
\hline LAWB99-1100-SSM-W & S-7517 & $<10.0$ & $<10.0$ \\
\hline LAWB99-1100-SSM-W & S-7517 & $<10.0$ & $<10.0$ \\
\hline LAWB99-1200-SSM-W & S-7518 & $<10.0$ & $<10.0$ \\
\hline LAWB99-1200-SSM-W & S-7518 & $<10.0$ & $<10.0$ \\
\hline 161SAS-950-SSM-W & S-7519 & $<10.0$ & $<10.0$ \\
\hline 161SAS-950-SSM-W & S-7519 & $<10.0$ & $<10.0$ \\
\hline 161SAS-1000-SSM-W & S-7520 & $<10.0$ & $<10.0$ \\
\hline 161SAS-1000-SSM-W & S-7520 & $<10.0$ & $<10.0$ \\
\hline 161SAS-1100-SSM-W & S-7521 & $<10.0$ & $<10.0$ \\
\hline 161SAS-1100-SSM-W & S-7521 & $<10.0$ & $<10.0$ \\
\hline 161SAS-1200-SSM-W & S-7522 & $<10.0$ & $<10.0$ \\
\hline 161SAS-1200-SSM-W & S-7522 & $<10.0$ & $<10.0$ \\
\hline 1ppm standard & - & 1.06 & 1.01 \\
\hline 5ppm standard & - & 5.34 & 5.22 \\
\hline LAWA161-950-SSM-W2 & S-7570 & $<10.0$ & $<10.0$ \\
\hline LAWA161-950-SSM-W2 & S-7570 & $<10.0$ & $<10.0$ \\
\hline \hline
\end{tabular}


Table B-4. ICP-MS Measurement Data for Cesium Concentrations in the Study Glass Wash Solutions

\begin{tabular}{|c|c|c|c|c|}
\hline \hline Sample ID & LAWA161-950-SSM-S & LAWA161-1000-SSM-S & LAWA161-1100-SSM-S & LAWA161-1200-SSM-S \\
\hline Lab ID & LW9931 S-7507 & LW9932 S-7508 & LW9933 S-7509 & LW9934 S-7510 \\
\hline Cs (mg/L) & 0.110 & 1.38 & 7.64 & 7.53 \\
\hline
\end{tabular}


Table B-5. Summary of Measured Composition Data (mg/L) for the Study Glass Wash Solutions

\begin{tabular}{|c|c|c|c|c|c|c|c|c|c|c|c|c|c|c|c|c|c|c|c|c|c|c|}
\hline Identifier & Al & B & $\mathrm{Ca}$ & $\mathrm{Cl}^{-}$ & $\mathrm{Cr}$ & Cs & $\mathbf{F}^{-}$ & $\mathbf{F e}$ & $\mathbf{K}$ & $\mathrm{Li}$ & Mg & $\mathrm{Na}$ & $\mathrm{Ni}$ & $\mathbf{P}$ & $\mathbf{P b}$ & $\mathrm{s}$ & Si & Sn & Ti & $\mathbf{v}$ & Zn & $\mathrm{Zr}$ \\
\hline LAWA161-950-SSM-W & $<1.00$ & 5.80 & $<1.00$ & $<10.0$ & $<1.00$ & 0.110 & $<10.0$ & $<1.00$ & $<0.500$ & $<1.00$ & $<1.00$ & 45.7 & $<1.00$ & $<2.00$ & $<1.00$ & 9.51 & 2.33 & $<1.00$ & $<1.00$ & 1.02 & $<1.00$ & $<1.00$ \\
\hline LAWA161-950-SSM-W2 & $<1.00$ & 6.09 & $<1.00$ & $<10.0$ & $<1.00$ & - & $<10.0$ & $<1.00$ & $<0.500$ & - & $<1.00$ & 48.6 & - & $<2.00$ & - & 9.12 & 3.57 & - & - & $<1.00$ & $<1.00$ & $<1.00$ \\
\hline LAWA161-1000-SSM-W & $<1.00$ & 9.94 & 3.17 & 11.1 & $<1.00$ & 1.38 & $<10.0$ & $<1.00$ & 5.87 & $<1.00$ & $<1.00$ & 259 & $<1.00$ & $<2.00$ & $<1.00$ & 168 & 2.43 & $<1.00$ & $<1.00$ & 2.48 & $<1.00$ & $<1.00$ \\
\hline LAWA161-1100-SSM-W & $<1.00$ & 24.2 & 18.7 & 113 & $<1.00$ & 7.64 & $<10.0$ & $<1.00$ & 27.2 & $<1.00$ & $<1.00$ & 923 & $<1.00$ & $<2.00$ & $<1.00$ & 572 & 2.53 & $<1.00$ & $<1.00$ & 6.31 & $<1.00$ & $<1.00$ \\
\hline LAWA161-1200-SSM-W & $<1.00$ & 26.9 & 19.0 & 108 & 1.19 & 7.53 & $<10.0$ & $<1.00$ & 25.6 & $<1.00$ & $<1.00$ & 828 & $<1.00$ & $<2.00$ & $<1.00$ & 519 & 1.76 & $<1.00$ & $<1.00$ & 5.98 & $<1.00$ & $<1.00$ \\
\hline ORPLG27-950-SSM-W & $<1.00$ & 12.7 & $<1.00$ & 10.3 & 36.2 & - & $<10.0$ & $<1.00$ & 201 & $<1.00$ & $<1.00$ & 487 & $<1.00$ & 2.08 & $<1.00$ & 361 & 5.53 & $<1.00$ & $<1.00$ & $<1.00$ & $<1.00$ & $<1.00$ \\
\hline ORPLG27-1000-SSM-W & $<1.00$ & 17.8 & $<1.00$ & 12.1 & 58.6 & - & $<10.0$ & $<1.00$ & 289 & $<1.00$ & $<1.00$ & 686 & $<1.00$ & 3.65 & $<1.00$ & 510 & 7.64 & $<1.00$ & $<1.00$ & $<1.00$ & $<1.00$ & $<1.00$ \\
\hline ORPLG27-1100-SSM-W & $<1.00$ & 22.0 & $<1.00$ & 16.9 & 53.8 & - & $<10.0$ & $<1.00$ & 355 & $<1.00$ & $<1.00$ & 821 & $<1.00$ & 3.95 & $<1.00$ & 603 & 2.98 & $<1.00$ & $<1.00$ & $<1.00$ & $<1.00$ & $<1.00$ \\
\hline ORPLG27-1200-SSM-W & $<1.00$ & 30.5 & $<1.00$ & 30.9 & 55.6 & - & $<10.0$ & $<1.00$ & 372 & $<1.00$ & $<1.00$ & 898 & $<1.00$ & 3.96 & $<1.00$ & 672 & 4.49 & $<1.00$ & $<1.00$ & $<1.00$ & $<1.00$ & $<1.00$ \\
\hline LAWB99-950-SSM-W & $<1.00$ & 6.62 & 72.8 & $<10.0$ & 5.27 & - & $<10.0$ & $<1.00$ & 23.1 & 45.1 & $<1.00$ & 641 & $<1.00$ & $<2.00$ & $<1.00$ & 613 & $<1.00$ & $<1.00$ & $<1.00$ & 5.46 & $<1.00$ & $<1.00$ \\
\hline LAWB99-1000-SSM-W & $<1.00$ & 6.45 & 74.4 & $<10.0$ & 3.86 & - & $<10.0$ & $<1.00$ & 24.3 & 46.9 & $<1.00$ & 614 & $<1.00$ & $<2.00$ & $<1.00$ & 593 & $<1.00$ & $<1.00$ & $<1.00$ & 5.25 & $<1.00$ & $<1.00$ \\
\hline LAWB99-1100-SSM-W & $<1.00$ & 7.75 & 69.1 & $<10.0$ & 3.18 & - & $<10.0$ & $<1.00$ & 24.1 & 41.7 & $<1.00$ & 517 & $<1.00$ & $<2.00$ & $<1.00$ & 527 & $<1.00$ & $<1.00$ & $<1.00$ & 5.07 & $<1.00$ & $<1.00$ \\
\hline LAWB99-1200-SSM-W & $<1.00$ & 11.3 & 58.3 & $<10.0$ & 2.78 & - & $<10.0$ & $<1.00$ & 25.4 & 38.4 & $<1.00$ & 515 & $<1.00$ & $<2.00$ & $<1.00$ & 485 & $<1.00$ & $<1.00$ & $<1.00$ & 5.02 & $<1.00$ & $<1.00$ \\
\hline 161SAS-950-SSM-W & $<1.00$ & 15.7 & 7.83 & $<10.0$ & $<1.00$ & - & $<10.0$ & $<1.00$ & 1.03 & $<1.00$ & $<1.00$ & 623 & $<1.00$ & $<2.00$ & $<1.00$ & 430 & 3.10 & $<1.00$ & $<1.00$ & $<1.00$ & $<1.00$ & $<1.00$ \\
\hline 161SAS-1000-SSM-W & $<1.00$ & 17.0 & 10.3 & $<10.0$ & $<1.00$ & - & $<10.0$ & $<1.00$ & 1.28 & $<1.00$ & $<1.00$ & 799 & $<1.00$ & $<2.00$ & $<1.00$ & 502 & 5.24 & $<1.00$ & $<1.00$ & $<1.00$ & $<1.00$ & $<1.00$ \\
\hline 161SAS-1100-SSM-W & $<1.00$ & 22.3 & 13.3 & $<10.0$ & $<1.00$ & - & $<10.0$ & $<1.00$ & 1.18 & $<1.00$ & $<1.00$ & 743 & $<1.00$ & $<2.00$ & $<1.00$ & 482 & 2.68 & $<1.00$ & $<1.00$ & $<1.00$ & $<1.00$ & $<1.00$ \\
\hline 161SAS-1200-SSM-W & $<1.00$ & 23.1 & 13.3 & $<10.0$ & $<1.00$ & - & $<10.0$ & $<1.00$ & 1.16 & $<1.00$ & $<1.00$ & 656 & $<1.00$ & $<2.00$ & $<1.00$ & 456 & 1.40 & $<1.00$ & $<1.00$ & $<1.00$ & $<1.00$ & $<1.00$ \\
\hline
\end{tabular}




\section{Distribution:}

J. W. Amoroso, 999-W

T. B. Brown, 773-A

M. E. Caldwell, 999-W

A. D. Cozzi, 999-W

C. L. Crawford, 773-42A

D. E. Dooley, 773-A

W. C. Eaton, PNNL

T. B. Edwards, 999-W

A. P. Fellinger, 773-42A

S. D. Fink, 773-A

K. M. Fox, 999-W

C. C. Herman, 773-A

A. M. Howe, 999-W

C. M. Jantzen, 773-A

T. Jin, PNNL

F. C. Johnson, 999-W

D. S. Kim, PNNL

A. A. Kruger, DOE-ORP

J. Matyáš, PNNL

D. J. McCabe, 773-42A

D. L. McClane, 999-W

G. A. Morgan, 999-W

F. M. Pennebaker, 773-42A

A. A. Ramsey, 999-W

W. G. Ramsey, 999-W

W. T. Riley, 999-1W

R. L. Russell, PNNL

M. J. Schweiger, PNNL

G. N. Smoland, 999-1W

C. L. Trivelpiece, 999-W

J. D. Vienna, PNNL

B. J. Wiedenman, 773-42A

W. R. Wilmarth, 773-A

Records Administration (EDWS) 